\title{
Moment searching algorithm for bioluminescence tomography
}

\author{
Ludong Jin (靳露冬) $)^{1}$, Yan Wu (吴 艳 $)^{1 *}$, Jie Tian (田 捷) $)^{1,2}$, \\ Heyu Huang (黄鹤羽) $)^{1}$, and Xiaochao Qu (屈晓超 $)^{1}$ \\ ${ }^{1}$ Life Science Research Center, School of Electronic Engineering, Xidian University, \\ Xi'an 710071, China \\ ${ }^{2}$ Medical Image Processing Group, Institute of Automation, Chinese Academy of Sciences, \\ Beijing 100190, China \\ *E-mail: ywu@mail.xidian.edu.cn \\ Received September 16, 2008
}

\begin{abstract}
To avoid the ill-posedness in the inverse problem of bioluminescence tomography, a moment searching algorithm fusing the finite element method (FEM) with the moment concept in theoretical mechanics is developed. In the algorithm, the source's information is mapped to the surface photon flux density by FEM, and the source's position is modified with the feedback through the algorithm of barycenter searching, which makes full use of the position information of the photon flux density on surface. The position is modified in every iterative step and will finally converge to the real source's value theoretically. OCIS codes: $170.3880,170.3890,170.3660,000.1430$.
\end{abstract}

doi: $10.3788 / C O L 20090707.0614$.

Molecular imaging will play an important role in future medical practice, efficaciously employed to detect and guide treatment of a disease at a stage before debilitating or irreversible symptoms appear ${ }^{[1]}$. Being a branch of the molecular imaging, bioluminescence tomography (BLT) has been attracting more attention due to its low equipment cost and high signal-to-noise ratio (SNR) in detection $^{[2]}$. In BLT, molecular and cellular events can be detected in vivo through the optical signals collected on body surface and both forward and inverse problems are involved, the aims of which are to obtain the fluence distribution and the source distribution respectively. However, the inverse problem is ill-posed ${ }^{[3]}$. The finite element method (FEM) and the adaptive finite element algorithm have been employed in $\mathrm{BLT}^{[4,5]}$, but they do not make the position information of photon flux density on surface used fully. In this letter, we develop a moment searching algorithm that fuses the FEM with the moment concept in theoretical mechanics, making the known position information utilized fully and the ill-posedness of the BLT reduced. The simulation results demonstrate the effectiveness of the algorithm.

When carried out in a dark environment, the BLT is modeled as the steady-state diffusion equation with Robin boundary condition mathematically ${ }^{[6]}$ :

$$
-\nabla \cdot(D(\mathbf{r}) \nabla \Phi(\mathbf{r}))+\mu_{\mathrm{a}}(\mathbf{r}) \Phi(\mathbf{r})=S(\mathbf{r}), \mathbf{r} \in \Omega,
$$

$\Phi(\mathbf{r})+2 A\left(n_{\mathrm{med}}, n_{\mathrm{env}}, \mathbf{r}\right) D(\mathbf{r})(\mathbf{v} \cdot \nabla \Phi(\mathbf{r}))=0, \mathbf{r} \in \partial \Omega$,

where $\Omega$ and $\partial \Omega$ are the solution domain and its boundary respectively; $\Phi(\mathbf{r})$ is the photon flux density $\left(\mathrm{W} / \mathrm{mm}^{2}\right) ; \mu_{\mathrm{a}}(\mathbf{r})$ denotes the absorption coefficient $\left(\mathrm{mm}^{-1}\right) ; S(\mathbf{r})$ is the source power density $\left(\mathrm{W} / \mathrm{mm}^{3}\right)$; $D(\mathbf{r})=1 /\left(3\left(\mu_{\mathrm{a}}(\mathbf{r})+(1-g) \mu_{\mathrm{s}}(\mathbf{r})\right)\right)$ is the optical diffusion coefficient $(\mathrm{mm}), \mu_{\mathrm{s}}(\mathbf{r})$ is the scattering coefficient $\left(\mathrm{mm}^{-1}\right)$, and $g$ is the anisotropy parameter; $\mathbf{v}$ is the unit outer normal on $\partial \Omega$; Given the refractiveindex-mismatched boundary, $A\left(n_{\mathrm{med}}, n_{\mathrm{env}}, \mathbf{r}\right)$ can be approximately represented as

$$
A\left(n_{\mathrm{med}}, n_{\mathrm{env}}, \mathbf{r}\right)=\frac{1+R(\mathbf{r})}{1-R(\mathbf{r})},
$$

where $R(\mathbf{r}) \approx-1.4399 n^{-2}+0.7099 n^{-1}+0.6681+0.0636 n$ and $n=n_{\text {med }} / n_{\text {env }} ; n_{\text {med }}$ is the refractive index of the phantom medium and $n_{\mathrm{env}}$ is the refractive index of the environment medium.

In a BLT experiment, the measured quantity is the outgoing photon flux density on $\partial \Omega$ :

$$
Q(\mathbf{r})=-D(\mathbf{r})(\mathbf{v} \cdot \nabla \Phi(\mathbf{r}))=\frac{\Phi(\mathbf{r})}{2 A\left(n_{\mathrm{med}}, n_{\mathrm{env}}, \mathbf{r}\right)} .
$$

The forward problem in BLT can be represented by

$$
\Phi(\mathbf{r})=f(S(\mathbf{r})), \mathbf{r} \in \Omega .
$$

The other parameters including the optical and structure information of the tissues needed in solving Eq. (1) can be obtained through diffusion optical tomography $(\mathrm{DOT})^{[7,8]}$ and computer tomography $(\mathrm{CT})$. While the inverse problem is

$$
S(\mathbf{r})=f^{-1}\left(\Phi\left(\mathbf{r}^{\prime}\right)\right), \mathbf{r} \in \Omega, \mathbf{r}^{\prime} \in \partial \Omega .
$$

We solve the diffusion equation by the FEM. The weak form of Eq. (1) is ${ }^{[9,10]}$

$$
\begin{aligned}
& \int_{\Omega}\left(D(\mathbf{r})(\nabla \Phi(\mathbf{r})) \cdot\left(\nabla v(\mathbf{r})+\mu_{\mathrm{a}}(\mathbf{r}) \Phi(\mathbf{r}) v(\mathbf{r})\right) \mathrm{d} \Omega\right. \\
& +\int_{\partial \Omega} \frac{1}{2 A\left(n_{\mathrm{med}}, n_{\mathrm{env}}, \mathbf{r}\right)} \Phi(\mathbf{r}) v(\mathbf{r}) \mathrm{d} A \\
& =\int_{\Omega} S(\mathbf{r}) v(\mathbf{r}) d \Omega, \forall v(\mathbf{r}) \in H^{1}(\Omega),
\end{aligned}
$$


where $H^{1}(\Omega)$ is the Sobolev space. Following the Galerkin method ${ }^{[9,10]}, \Phi(\mathbf{r})$ and $v(\mathbf{r})$ have the same function space and $\Phi(\mathbf{r})$ is approximated as

$$
\Phi(\mathbf{r}) \approx \sum_{i=1}^{N_{\text {node }}} \phi_{i} \varphi_{i}^{\text {node }}(\mathbf{r})
$$

where $\phi_{i}$ is the value of the $i$ th node; $\varphi_{i}^{\text {node }}(\mathbf{r})$ is the node's basis function and $N_{\text {node }}$ is its grid number. At the same time, $S(\mathbf{r})$ is discretized on the same finite element mesh as

$$
S(\mathbf{r}) \approx \sum_{j=1}^{N_{\text {elem }}} s_{j} \varphi_{j}^{\text {elem }}(\mathbf{r}),
$$

where $s_{j}$ is the $j$ th element's value and $\varphi_{j}^{\text {elem }}(\mathbf{r})$ is the basis function defined on this element; $\mathrm{N}_{\text {elem }}$ is the total element number. The reason why the element rather than the node is the unit used in the discretization of $S(\mathbf{r})$ is that the element is the smallest volume unit, which makes the algorithm more sensitive in searching for the source, while a node's value depends on the values of the elements connected with it.

Substituting Eqs. (8) and (9) into Eq. (7), we obtain

$$
K \Phi=F S,
$$

where $K=\left[k_{i j}\right]_{N_{\text {node }} \times N_{\text {node }}}, \Phi=\left[\phi_{i}\right]_{N_{\text {node }} \times 1}, F=$ $\left[f_{i j}\right]_{N_{\text {node }} \times N_{\text {elem }}}, S=\left[s_{i}\right]_{N_{\text {elem }} \times 1}$, and

$$
\begin{aligned}
k_{i j} & =\int_{\Omega} D(\mathbf{r})\left(\nabla \varphi_{i}^{\text {node }}(\mathbf{r})\right) \cdot\left(\nabla \varphi_{j}^{\text {node }}(\mathbf{r})\right) \mathrm{d} \Omega \\
& +\int_{\Omega} \mu_{a}(\mathbf{r}) \varphi_{i}^{\text {node }}(\mathbf{r}) \varphi_{j}^{\text {node }}(\mathbf{r}) \mathrm{d} \Omega \\
& +\int_{\partial \Omega} \frac{1}{\left.2 A_{(} n_{\text {med }}, n_{\mathrm{env}}, \mathbf{r}\right)} \varphi_{i}^{\text {node }}(\mathbf{r}) \varphi_{j}^{\text {node }}(\mathbf{r}) \mathrm{d} A \\
& f_{i j}=\int_{\Omega} \varphi_{i}^{\text {node }}(\mathbf{r}) \varphi_{j}^{\text {elem }}(\mathbf{r}) \mathrm{d} \Omega .
\end{aligned}
$$

Equation (10) expresses the relationship between the distribution of the photon flux density and the distribution of the source power density. However, for the photon flux density, the data we are interested in are on the body surface, which makes us rearrange Eq. (10) as

$$
\left[\begin{array}{l}
M_{11} M_{12} \\
M_{21} M_{22}
\end{array}\right]\left[\begin{array}{l}
\Phi_{\text {sur }} \\
\Phi_{\text {in }}
\end{array}\right]=\left[\begin{array}{l}
F_{1} \\
F_{2}
\end{array}\right] S,
$$

where $\Phi_{\text {sur }}$ is the photon flux density on surface and $\Phi_{\text {in }}$ is the internal photon flux density of the phantom. The sub-matrices, $M_{11}, M_{12}, M_{21}$, and $M_{22}$ of $M$, and $F_{1}$ and $F_{2}$ of $F$, are arranged in consistence with $\Phi_{\text {sur }}$ and $\Phi_{\text {in }}$.

Equation (11) can be evaluated as

$$
\begin{aligned}
\Phi_{\text {sur }}(S)= & \left(M_{11}-M_{12} M_{22}^{-1} M_{21}\right)^{-1} \\
& \left(F_{1}-M_{12} M_{22}^{-1} F_{2}\right) S .
\end{aligned}
$$

Then we can get the photon flux density on the surface from the source distribution directly through Eq. (12).

In the moment searching algorithm, the moment of the flux about the plane is defined as the product of the photon flux density and the corresponding coordinate value. For example, the moment of the flux about the yoz plane is $^{[11]}$

$$
M_{y o z}(\Phi)=\sum_{i=1}^{N_{\mathrm{sn}}} \Phi_{i} x_{i}
$$

where $N_{\mathrm{sn}}$ is the number of the surface node; $\Phi_{i}$ is the node value of the $i$ th node on the surface and $x_{\mathrm{i}}$ is its $x$ coordinate value. $M_{x o z}$ and $M_{x o y}$ are defined similarly.

According to the theory on finding the barycenter in theoretical mechanics, we can get a position as

$$
\left\{\begin{array}{l}
p x(\Phi)=M_{y o z}(\Phi) / \sum_{i=1}^{N_{\mathrm{sn}}} \Phi_{i} \\
p y(\Phi)=M_{x o z}(\Phi) / \sum_{i=1}^{N_{\mathrm{sn}}} \Phi_{i} . \\
p z(\Phi)=M_{x o y}(\Phi) / \sum_{i=1}^{N_{\mathrm{sn}}} \Phi_{i}
\end{array}\right.
$$

To search for the source, we give an initial guess of the source's position and the element enclosing the position is considered as the source. In other words, the elements of the matrix $S$ in Eq. (12) are zero except for the one which represents the source. At the same time, the optimum source power density is obtained through

$$
\min \left(\left\|\Phi_{\mathrm{sur}}(S)-\Phi_{\mathrm{m}}\right\|\right),
$$

where $\Phi_{\mathrm{m}}$ is the photon flux density measured on the body surface. Then we can give a feedback to the source's position through Eq. (14). Actually, if the guessed source's position deviates from the real source in a direction, the value of $\left\|\Phi_{\text {sur }}-\Phi_{\mathrm{m}}\right\|$ in this direction is larger than the other directions, according to which the guessed position is adjusted. This algorithm makes the position information used fully. The next position is obtained as

$$
\begin{gathered}
\left\{\begin{array}{l}
x_{i+1}=x_{i}+\lambda\left(x_{m}-x_{i}\right) \\
y_{i+1}=y_{i}+\lambda\left(y_{m}-y_{i}\right) \\
z_{i+1}=z_{i}+\lambda\left(z_{m}-z_{i}\right)
\end{array}\right. \\
\left\{\begin{array}{l}
x_{m}=p x\left(\max \left(\Phi_{\text {sur }}-\Phi_{\mathrm{m}}\right)-\left(\Phi_{\mathrm{sur}}-\Phi_{\mathrm{m}}\right)\right) \\
y_{m}=p y\left(\max \left(\Phi_{\mathrm{sur}}-\Phi_{\mathrm{m}}\right)-\left(\Phi_{\mathrm{sur}}-\Phi_{\mathrm{m}}\right)\right) \\
z_{m}=p z\left(\max \left(\Phi_{\mathrm{sur}}-\Phi_{\mathrm{m}}\right)-\left(\Phi_{\mathrm{sur}}-\Phi_{\mathrm{m}}\right)\right)
\end{array}\right.
\end{gathered}
$$

where $\left(x_{i}, y_{i}, z_{i}\right)$ is the guessed source position in the $i$ th iteration, $0<\lambda<1$.

Equation (16) makes the source's position move toward the real one. This process is done again and again until the guessed position converges to a certain place. Compared with the theoretical mechanics, the surface point is corresponding to the particle. As the photon flux densities in all directions are equally important, we must configurate the phantom's surface points so that the surface points are symmetric about the $x, y$, and $z$ axes.

We have simulated the algorithm with a cylinder phantom of $30-\mathrm{mm}$ height and $10-\mathrm{mm}$ radius as shown in Fig. 1. Its optical parameters are shown in Table $1^{[5]}$. In the experiment, the initial position of the source is set 


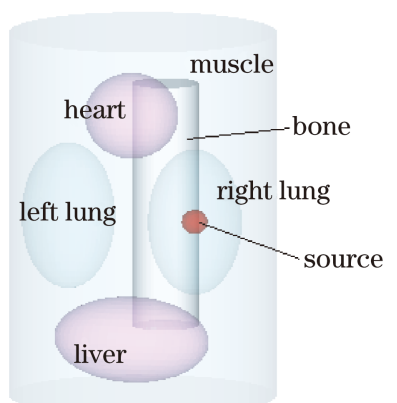

Fig. 1. Phantom used in the simulation composed of muscle, lungs, heart, bone, liver, and source.

Table 1. Optical Parameters of the Phantom in Fig. 1

\begin{tabular}{cccccc}
\hline & Muscle & Lung & Heart & Bone & Liver \\
\hline$\mu_{\mathrm{a}}\left(\mathrm{mm}^{-1}\right)$ & 0.010 & 0.350 & 0.200 & 0.002 & 0.035 \\
$\mu_{\mathrm{s}}\left(\mathrm{mm}^{-1}\right)$ & 4.0 & 23.0 & 16.0 & 20.0 & 6.0 \\
$g$ & 0.90 & 0.94 & 0.85 & 0.90 & 0.90 \\
\hline
\end{tabular}

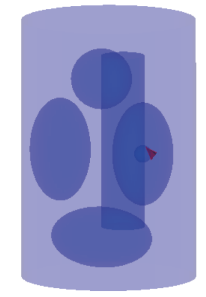

(a)

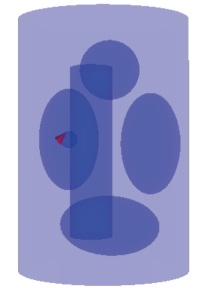

(c)

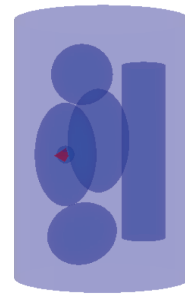

(b)

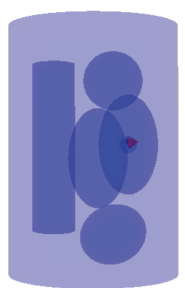

(d)
Fig. 2. Simulation results of (a) front view, (b) left view, (c) back view, and (d) right view. The dark elements represent the reconstructed sources.

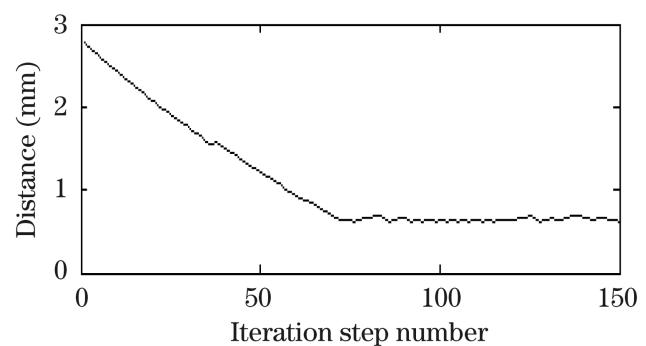

Fig. 3. Convergence curve of distance from the reconstructed source position to the real source on $y$-axis with the iteration step number on $x$-axis.

at $\left(p x\left(\Phi_{\mathrm{m}}\right), p y\left(\Phi_{\mathrm{m}}\right), p z\left(\Phi_{\mathrm{m}}\right)\right)$ and the simulation results are shown in Fig. 2, from which we can see that the reconstructed source's position coincides with the real one. Figure 3 shows the convergence curve of the distance between real source and reconstructed source with the iteration number where the position converges to the point $(2.276,5.577,-0.004), 0.61 \mathrm{~mm}$ away from the center of the real source's position $(2.500,5.000,0.000)$ after the 80th step. It demonstrates the accuracy of the algorithm in position searching when compared with the adaptive finite element algorithm which converges to a point $0.93 \mathrm{~mm}$ away from the real source ${ }^{[5]}$. According to the definition of power density, the total power of $1.12 \mathrm{nW}$ is obtained compared with the real source's power of $4.19 \mathrm{nW}$. From the results, we can see that the moment searching algorithm can obtain the position information effectively. But the power information is not good enough and needs further improvement.

In conclusion, this proposed algorithm fuses the FEM with the moment concept in theoretical mechanics. Enlighted by the algorithm in finding the barycenter, the position information of photon flux density is fully used. The simulation results show the effectiveness of the algorithm. However, the simulation composed of two or more sources is also needed and the algorithm should be further modified.

This work was supported by the program for Cheung Kong Scholars and Innovative Research Team in University of China (No. IRT0645), the National Natural Science Foundation of China (No. 60872137), the National Defence Foundation of China (No. 9140A01060408DZ0104), the National "973" Program of China (No. 2006CB705700), and the Program for Chair Professors of Cheung Kong Scholars Program of China.

\section{References}

1. K. Bi, X. Xu, L. Xi, S. Zeng, and Q. Luo, Chin. Opt. Lett. 6, 893 (2008).

2. A. Hengerer, A. Wunder, D. J. Wagenaar, A. H. Vija, M. Shah, and J. Grimm, Proc. IEEE 93, 819 (2005).

3. G. Wang, Y. Li, and M. Jiang, Med. Phys. 31, 2289 (2004).

4. W. Cong, G. Wang, D. Kumar, Y. Liu, M. Jiang, L. V. Wang, E. A. Hoffman, G. McLennan, P. B. McCray, J. Zabner, and A. Cong, Opt. Express 13, 6756 (2005).

5. Y. Lv, J. Tian, W. Cong, G. Wang, J. Luo, W. Yang, and H. Li, Opt. Express 14, 8211 (2006).

6. L. V. Wang and H. Wu, Biomedical Optics: Principles and Imaging (Wiley, New Jersey, 2007) pp.83-118.

7. Q. Zhang, L. Yin, Y. Tan, Z. Yuan, and H. Jiang, Opt. Express 16, 1481 (2008).

8. H. Niu, P. Guo, X. Song, and T. Jiang, Chin. Opt. Lett. 6, 886 (2008).

9. M. Wang and M. Shao, The Basic Principle and Numerical Methods of Finite Element Method (in Chinese) (Tsinghua University Press, Beijing, 1997) pp.12-20.

10. J. Lu and Z. Guan, The Numerical Solution of Partial Differential Equations (2nd edn.) (in Chinese) (Tsinghua University Press, Beijing, 2004) pp.187-210.

11. Z. Zhu, Q. Zhou, and J. Yin, Theoretical Mechanics (in Chinese) (Peking University Press, Beijing, 1997) pp.18-23. 